\title{
L'emodiafiltrazione ad alto flusso
}

\section{Leopoldo Cioni}

\author{
Divisione Nefrologica - Spedali Riuniti S. Chiara, Pisa
}

$\mathbf{L}$ insufficienza renale cronica evolve in un periodo di tempo più o meno lungo, ma inesorabilmente, verso l'uremia, che è quella condizione di assoluta abolizione della funzione renale. Questo stato è incompatibile con la vita, per cui dobbiamo ricorrere ad una terapia che sostituisca la funzione renale.

La terapia sostitutiva può essere artificiale (rene artificiale) o mediante trapianto renale.

Quanto detto è sintetizzato nel seguente schema:
L'argomento riguardante l'emodiafiltrazione (HDF) ad alto flusso fa parte della terapia sostitutiva extracorporea. Prima di trattarne dettagliatamente, ritengo che siano necessarie alcune premesse.

La sostituzione artificiale della funzione renale ha lo scopo di allontanare dall'organismo $\mathrm{i}$ liquidi non eliminati e le sostanze tossiche responsabili dell'uremia, oltre a risparmiare il più possibile i costituenti normali dell'organismo.

Come abbiamo visto nello schema, la terapia sostitutiva artificiale può essere intracorporea ed extracorporea ed è rappresentata da:

- Dialisi peritoneale (o emodialisi intracorporea)

- Emodialisi extracorporea (comunemente emodialisi)

- Emofiltrazione

- Emodiafiltrazione.

La dialisi peritoneale non fa parte della presente trattazione. È ca ritenersi fondamentale trattare sommariamente dei principi della emodialisi e della emofiltrazione, perché queste metodiche costituiscono la base della emodiafiltrazione.

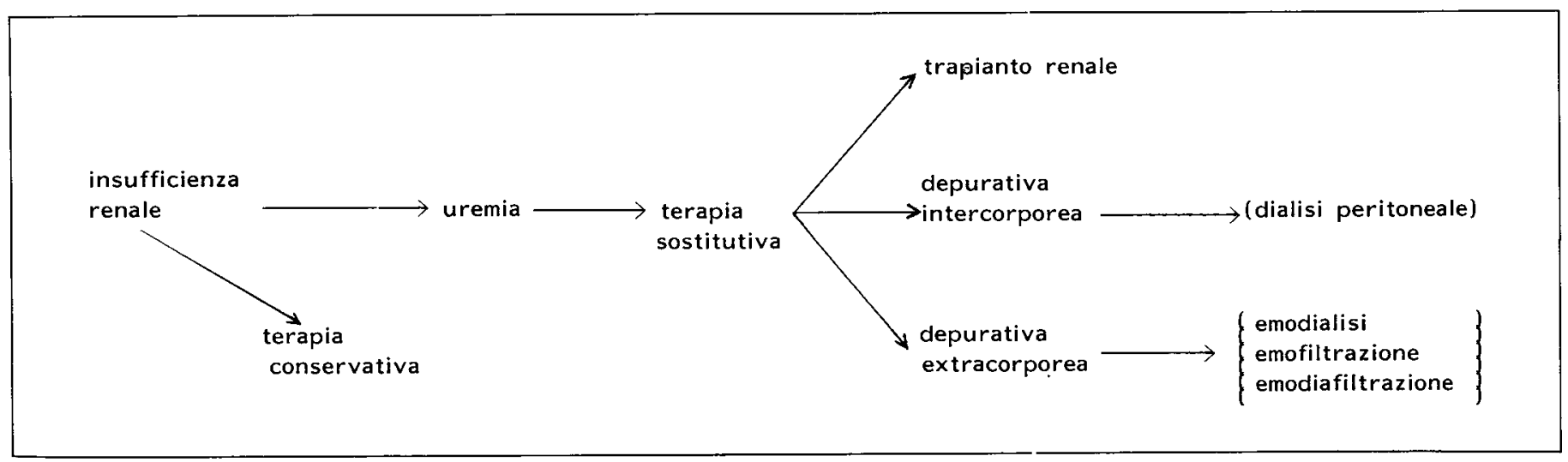




\section{Emodialisi}

Per emodialisi si intende il passaggio di soluti dal compartimento ematico a quello della soluzione elettrolitica attraverso membrane ad essi permeabili.

Il passaggio di soluti attraverso le membrane avviene per diffusione ed è in funzione del gradiente di concentrazione del soluto ai due lati della membrana (compartimento ematico e compartimento della soluzione elettrolitica), come è rappresentato nel seguente schema. (Fig. 1).

In piccola percentuale il passaggio di un soluto attraverso una membrana ad esso permeabile avviene anche mediante l'ultrafiltrazione per trascinamento del soluto da parte del solvente, provocato dalla eventuale pressione transmembrana. La pressione transmembrana è data dalla pressione positiva esercitata sul compartimento ematico, da quella negativa esercitata sulla soluzione elettrolitica o dalla somma (senza tener conto dei segni positivo e negativo) delle due pressioni (Esempio: positiva $+100 \mathrm{mmHg}$, negativa $-100 \mathrm{mmHg}, \mathrm{TMP}=200 \mathrm{mmHg}$ ) (Figg. 2, 3).

L'ultrafiltrazione ̀̀ necessaria quando il paziente ritiene liquido nel periodo interdialitico.

Dal punto di vista pratico, l'ultrafiltrazione si effettua secondo le modalità indicate nel seguente schema di emodialisi. (Fig. 4).

Quando, invece, vogliamo ridurre l'entità della ultrafiltrazione spontanea, possiamo controbilanciare la pressione positiva del compartimento ematico (Esempio: $+100 \mathrm{mmHg}$ ) con una pressione positiva del compartimento della

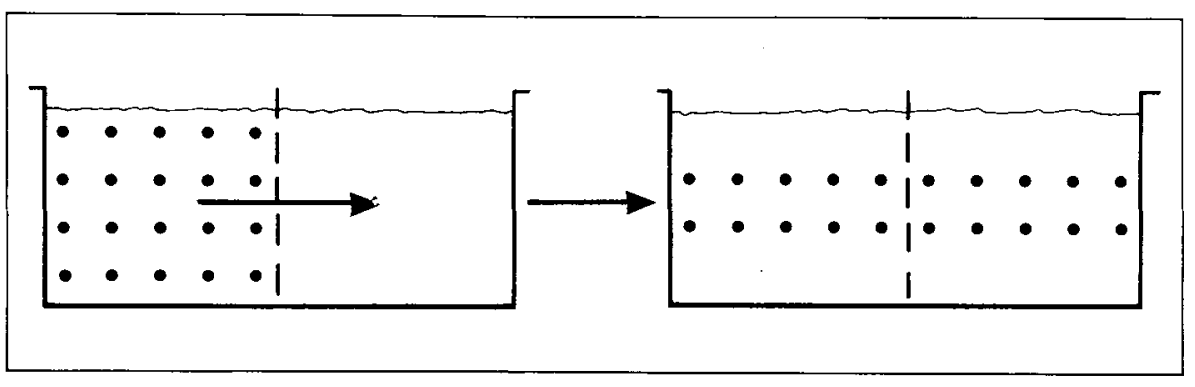

Fig. 1



Fig. 2 - Ultrafiltrazione per pressione positiva

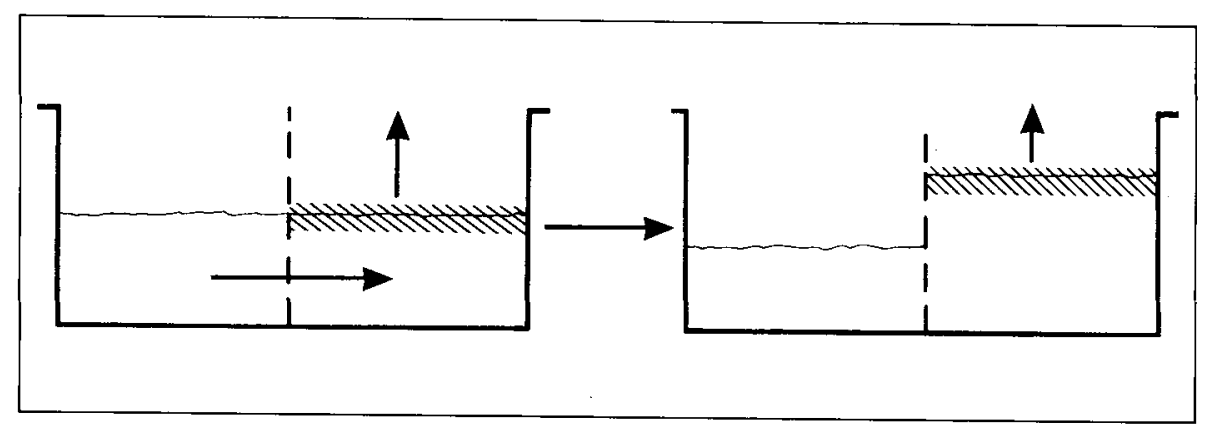

Fig. 3 - Ultrafiltrazione per pressione negativa

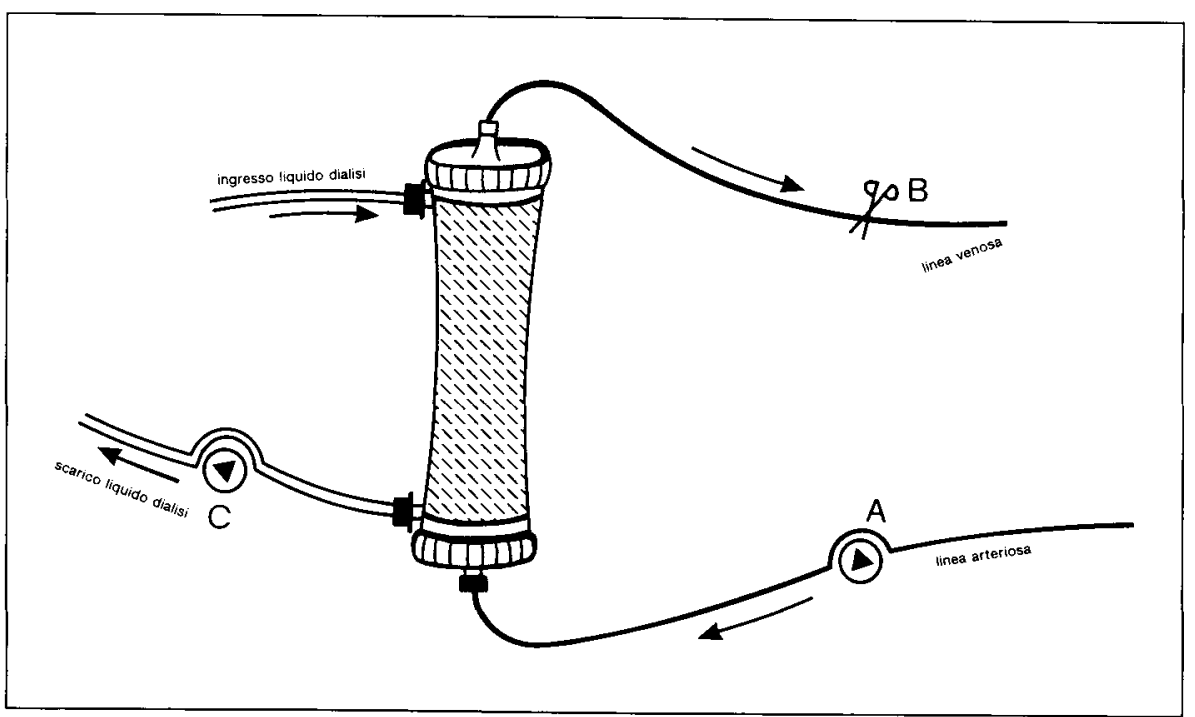

Fig. 4 - $A=$ pompa sangue (pressione positiva)

$B=$ morsetto che restringe la linea venosa sotto il gocciolatore (pressione positiva) $C=$ pompa aspirante il dialisato (pressione negativa) 


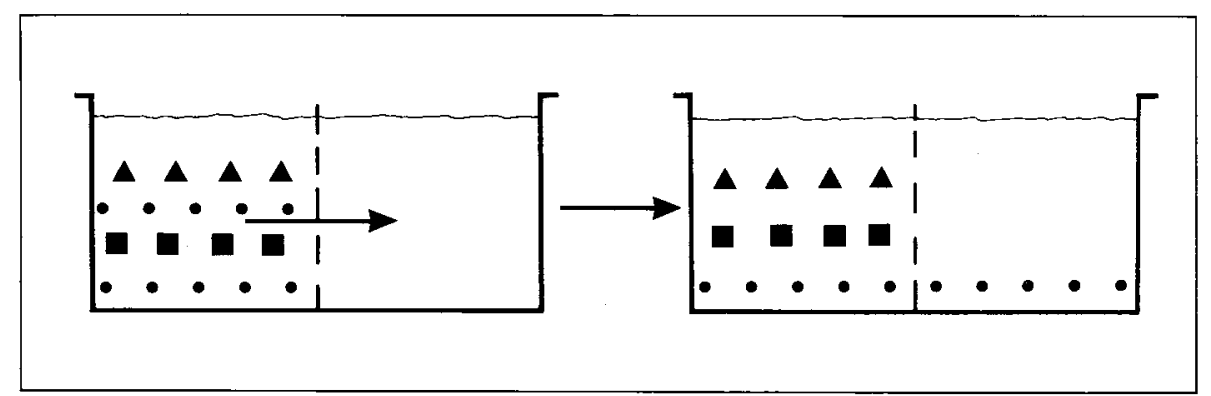

Fig. 5

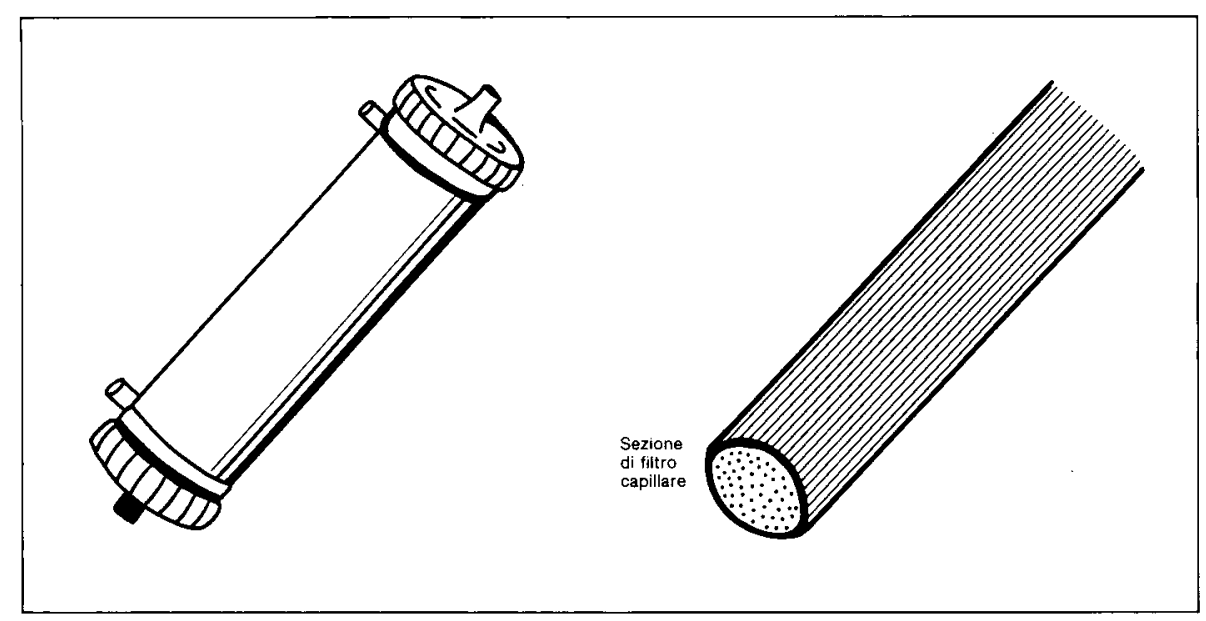

Fig. 6 - Filtro capillare

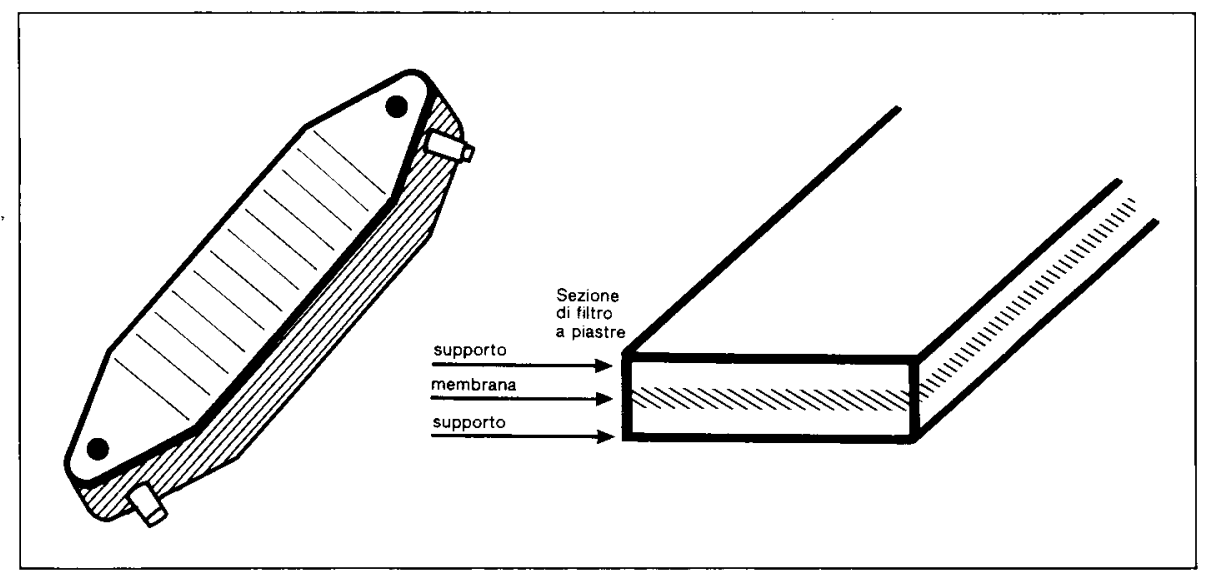

Fig. 7 - Filtro a piastre

soluzione elettrolitica (Esempio: $+50 \mathrm{~mm} \mathrm{Hg}$ ). Allora la pressione transmembrana non sarà $100+50$, ma $100-50=50 \mathrm{mmHg}$.

Le membrane che normalmente vengono usate per la emodialisi sono costituite da cuprofan o da materiale con le stesse caratteristiche. Esse sono porose con un diametro medio dei pori di 20-25 Ar- mstrong. Questi pori permettono il passaggio di soluti che hanno un peso molecolare non superiore ai 600 Daltons, come si può osservare nello schema seguente (Fig. 5), dove i punti rappresentano i soluti che attraversano la membrana, mentre $\Delta$ e $\square$ quelli di peso molecolare superiore ai 600 Daltons, che non attraversano la membrana.

Con l'emodialisi è quindi possibile allontanare dall'organismo solo i soluti tossici di piccolo peso molecolare insieme all'acqua, come abbiamo visto parlando della ultrafiltrazione.

Tuttavia, oggi sono numerosi $\mathrm{i}$ pazienti in emodialisi da oltre 20 anni in buone condizioni soggettive e di riabilitazione, per cui si può affermare che fra le piccole molecole ci sono sostanze note (Esempio: urea, creatinina) ed ignote che hanno una importanza dominante nel determinare la sindrome uremica.

Essa regredisce completamente quando la emodialisi viene eseguita in maniera adeguata.

I filtri usati per la emodialisi possono essere a capillari o a piastre (il materiale dializzante è sempre costituito da cuprofan o da materiali con le stesse caratteristiche di porosità). Attualmente sono in commercio anche filtri con membrane a più alta porosità. Lo schema seguente mostra i 2 tipi di filtri (Figg. 6, 7).

Naturalmente, i capillari di un filtro sono numerosi, come pure nei filtri a piastra $i$ compartimenti ematici sono parecchi. All'interno dei capillari circola il sangue ed al loro esterno la soluzione elettrolitica.

In quelli a piastre abbiamo: supporto, membrana dializzante, 
membrana dializzante, supporto e così via. Fra le 2 membrane circola il sangue; fra la membrana e il supporto circola la soluzione elettrolitica.

\section{Emofiltrazione}

Come abbiamo detto, con la emodialisi si depura l'organismo soltanto dei soluti tossici di piccolo peso molecolare. Invece, il glomerulo umano non fa distinzione fra piccole e medie molecole perché lascia passare, seppure in misura via via più ridotta, anche grosse molecole fino ad una certa quantità di albumina, che ha un peso molecolare di 63.000 Daltons. Infatti, la misura più esatta del filtrato glomerulare ci è fornita dalla clearance dell'inulina, sostanza dal peso molecolare di 5.200 Daltons, che viene filtrata dal glomerulo come la creatinina, che ha un peso molecolare di 113 Daltons. Il passaggio delle sostanze filtrabili attraverso la membrana basale del glomerulo non avviene per diffusione, ma per pressione idrostatica con conseguente trasporto convettivo con l'acqua in cui sono disciolte. La emofiltrazione è quella metodica con la quale vengono utilizzate membrane ad elevata permeabilità idraulica che consentono il passaggio anche di soluti del peso molecolare superiore ai 20.000 Daltons. La permeabilità all'acqua di queste membrane, fra cui le più usate sono quelle di poliacrilonitrile, polimetilmetacrilato, polisulfone, poliammide, è circa 40 volte superiore a quella offerta dal cuprofan o suoi derivati. Nella emofiltrazione le membrane ad elevata permeabilità i-

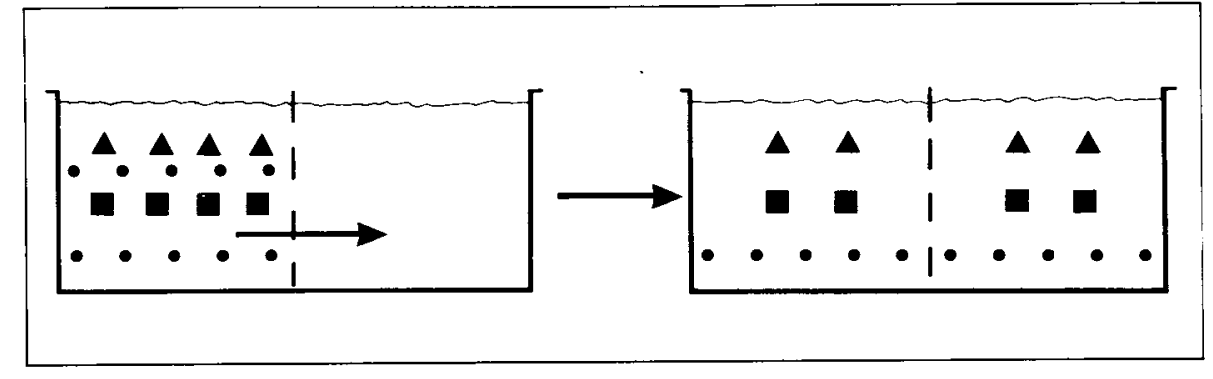

Fig. 8

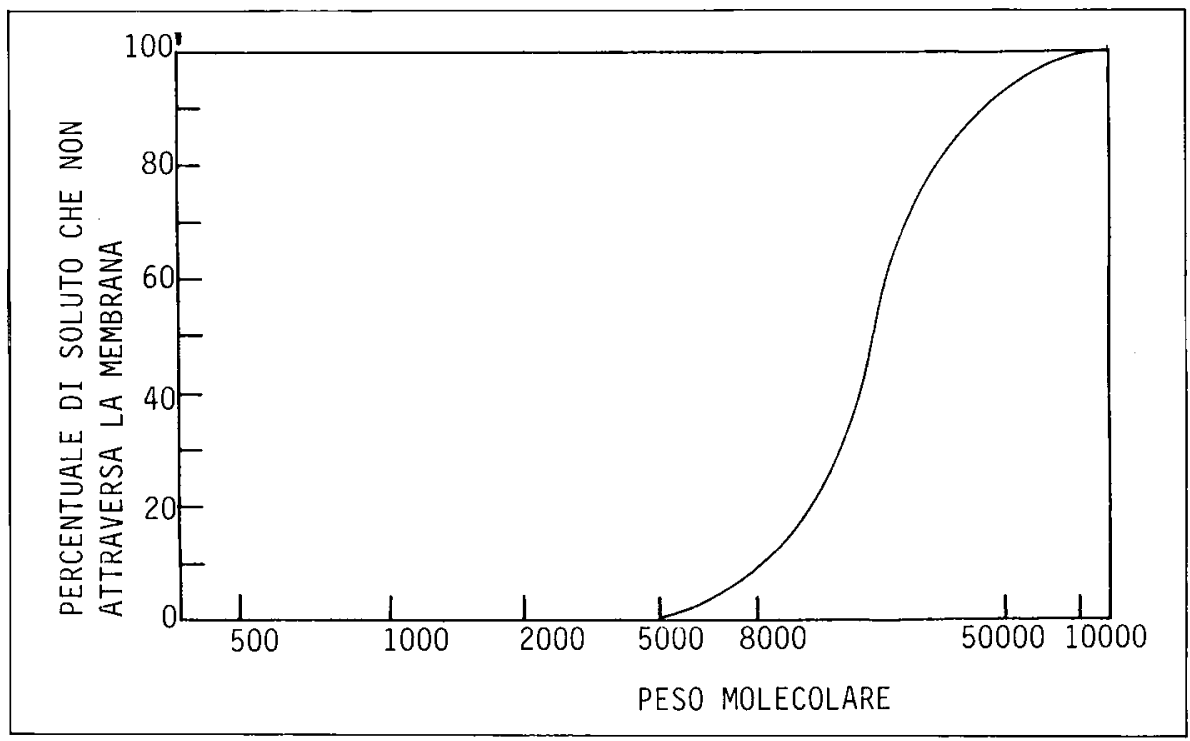

Fig. 9 - Percentuale di soluto che non attraversa la membrana

draulica vengono utilizzate nella maniera più simile alla funzione del glomerulo, cioé senza che sulla faccia della membrana opposta a quella del compartimento ematico circoli la soluzione elettrolitica, ma solo esercitando una pressione positiva sul compartimento ematico e, se necessario, anche una pressione negativa all'uscita dell'emofiltrato.

L'emofiltrato è rappresentato dall'acqua plasmatica e da quei soluti che hanno attraversato la membrana per trasporto convettivo.

Lo schema rappresenta il passaggio dei soluti con la emofiltra- zione. (Fig. 8).

Si può notare che con la emofiltrazione passano anche $i$ soluti di elevato peso molecolare.

Le varie membrane ad elevata permeabilità sono simili fra loro dal punto di vista funzionale e si può dire che con esse non esistono differenze apprezzabili del coefficiente di filtrazione fra una piccola molecola ed una media molecola del peso dell'inulina.

Le differenze cominciano con molecole del peso di oltre 8.000 Daltons, fino ad aversi un coefficiente di filtrazione vicino a zero per pesi molecolari superiori a 40.000 Daltons. 


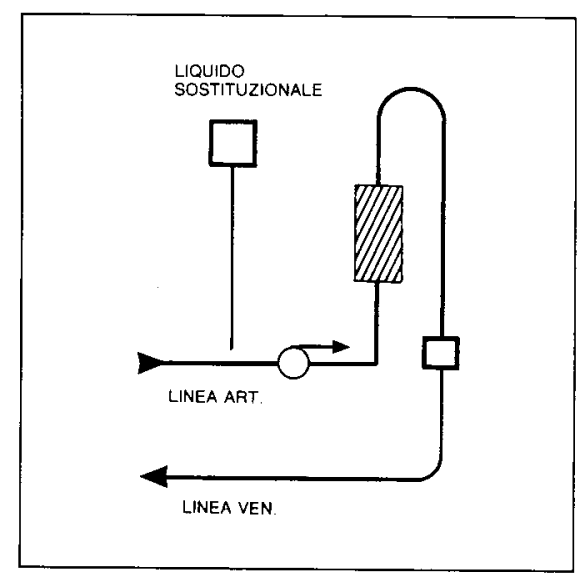

Fig. 10 - Somministrazione del liquido di sostituzione con la metodica della prediluizione

Il coefficiente di filtrazione esprime la misura della porosità della membrana ed è dato dal rapporto tra la concentrazione del soluto nell'emofiltrato e quello del soluto nell'acqua plasmatica.

Quando un soluto passa totalmente si ha un coefficiente di filtrazione uguale a 1.

La Figura 9 rappresenta il comportamento del soluto verso la membrana ad elevata permeabilità idraulica in funzione del suo peso molecolare.

Per effettuare l'emofiltrazione vengono utilizzati filtri capillari di superficie compresa fra $\mathrm{m}^{2} 1.6 \mathrm{e} \mathrm{m}^{2}$ 2.1 che, con un flusso ematico di $400-500 \mathrm{ml} / \mathrm{min}$ e con una pressione transmembrana di $400 \mathrm{mmHg}$, possono fornire un emofiltrato orario di 8-10 litri (circa $\mathrm{ml} 15 / \mathrm{h}$ / $\mathrm{mmHg} / \mathrm{m}^{2}$ ).

L'emofiltrato rappresenta la funzione del glomerulo umano e la sua quantità nell'unità di tempo è molto elevata.

Per questo motivo, dobbiamo contemporaneamente sostituire la funzione renale tubulare reintegrando al paziente la quantità di liquido e di elettroliti che via via

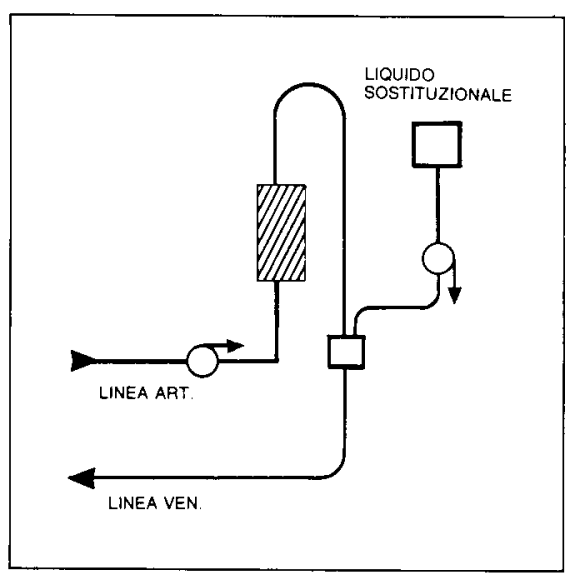

Fig. 11 -Somministrazione del liquido di sostituzione con la metodica della postdiluizione

vengono filtrati in eccesso. A questo scopo viene continuamente somministrata al paziente una soluzione elettrolitica che, salvo variazioni apportate dai singoli operatori, ha la seguente composizio-

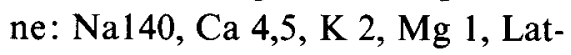
tato $42, \mathrm{Cl} 106 \mathrm{mEq} / 1$ con gr $1 \%$ o $2 \%$ di glucosio. L'osmolarità di questo liquido di sostituzione è compresa tra $299 \mathrm{mOsm} / 1$ (Glucosio $1 \%$ ) e $304 \mathrm{mOsm} / 1$ (glucosio $2 \% 0$ ). La quantità di liquido che deve essere somministrata durante il trattamento è uguale alla quantità di emofiltrato meno la perdita di peso programmata. La somministrazione può essere effettuata secondo la tecnica della prediluizione o quella della postdiluizione (nella linea arteriosa nel primo caso e nella linea venosa nel secondo), come è rappresentato nelle figure (Figg. 10, 11).

Può essere effettuata anche una somministrazione combinata. Quando il liquido di sostituzione viene somministrato in post-diluizione deve essere precedentemente riscaldato in termostato a $37^{\circ} \mathrm{C}$.

I monitors per emofiltrazione sono differenti da quelli per emo- dialisi. Sono tutti in grado di operare le manovre suddette; devono soltanto essere programmati.

\section{Clearances}

Prima di trattare della emodiafiltrazione, è opportuno sintetizzare ora gli effetti funzionali della emodialisi e della emofiltrazione. Ciò faciliterà notevolmente la trattazione della emodiafiltrazione.

I filtri con membrana di cuprofan o di materiale con le stesse caratteristiche fisiche, hanno la capacità di depurare una certa quantità di sangue nell'unità di tempo mediante una depurazione diffusiva (o clearance diffusiva), che è scarsamente influenzata dalla pressione transmembrana per la scarsa permeabilità del filtro.

La clearance diffusiva è in stretta relazione con: flusso ematico, flusso della soluzione elettrolitica, superficie del filtro.

I filtri con membrana ad elevata permeabilità idraulica, che vengono usati per emofiltrazione hanno anche la capacità di depurare una certa quantità di sangue nell'unità di tempo mediante una depurazione convettiva (o clearance convettiva), che è notevolmente influenzata dalla pressione transmembrana per la elevata permeabilità del filtro.

La clearance convettiva dipende da: flusso ematico, pressione transmembrana, superficie del filtro.

In emofiltrazione la clearance convettiva si può identificare con la quantità di emofiltrato per minuto, almeno fino a pesi molecolari dell'ordine di 5.000 Daltons, dipendendo dal coefficiente di filtrazione del soluto, come indicato nella seguente Tabella I. 
TAB. I

\begin{tabular}{lcc}
\hline Soluto & Peso molecolare & $\begin{array}{c}\text { Coefficiente } \\
\text { di filtrazione }\end{array}$ \\
\hline Urea & 60 & 1.0 \\
\hline Vit. B 12 & 1.355 & 1.0 \\
\hline Inulina & 5.200 & 0.99 \\
\hline Albumina & 63.000 & 0.05 \\
\hline
\end{tabular}

Se noi usiamo un filtro per emofiltrazione con la soluzione elettrolitica e quindi con le stesse modalità di un filtro per emodialisi, otteniamo quella metodica che è denominata modiafiltrazione.

Con l'emodiafiltrazione si sommano la clearance diffusiva della emodialisi e la clearance convettiva della emofiltrazione. Pertanto, tale metodica è capace di sommare gli effetti positivi della emodialisi a quelli della emofiltrazione, di ridurre anche notevolmente il tempo di trattamento proprio per la somma delle clearances e di assicurare il pieno benessere del paziente. Se praticata in maniera adeguata, l'emodiafiltrazione è da considerarsi attualmente la metodica che consente il più completo sfruttamento delle membrane ad elevata permeabilità idraulica. Per ottenere che le clearances (diffusiva e convettiva) siano molto elevate, bisogna utilizzare quei parametri che sono già stati indicati e cioè:

- Elevato flusso ematico

- Elevato flusso della soluzione elettrolitica

- Elevata pressione transmembrana

- Elevata superficie del filtro

È possibile praticare una emodiafiltrazione che si può denominare soft (leggera-dolce), con filtri di modesta superficie e flussi non troppo elevati, ma questa non è oggetto della presente trattazione.
L'emodiafiltrazione che viene qui trattata si può invece denominare hard (spinta-forte), con la quale vengono sfruttati i filtri $\mathrm{di}$ ampia superficie.

Per praticare la emodiafiltrazione ad alto flusso devono essere seguite le seguenti modalità tecniche:

- Flusso ematico $\left(\mathrm{Q}_{\mathrm{b}}\right)$ uguale o superiore a $450 \mathrm{ml} / \mathrm{min}$

- Flusso della soluzione elettrolitica $\left(Q_{d}\right)$ uguale o superiore a $700 \mathrm{ml} / \mathrm{min}$

- Pressione transmembrana (TMP) di 400-500 $\mathrm{mmHg}$

- Un filtro che con i parametri suddetti di $\mathbf{Q}_{b}$ e $\mathbf{Q}_{d}$ consente una clearance dell'urea superiore ai $300 \mathrm{ml} / \mathrm{min}$.

Nella seguente Tabella sono indicati i filtri particolarmente idonei a praticare l'emodiafiltrazione ad alto flusso (Tab. II).

Dal punto di vista pratico, l'emodiafiltrazione ad alto flusso si pratica come una comune emodialisi con la differenza che, durante il trattamento, dovremo restituire al paziente quella quantità di liquido di sostituzione che è uguale all'emofiltrato meno la perdita di peso programmata.

Il liquido di sostituzione è ugua- le a quello che viene utilizzato per la emofiltrazione e viene in genere somministrato con la metodica della postdiluizione.

Per quanto riguarda il tipo di monitor, viene utilizzato un normale monitor per emodialisi, dotato di pompa sangue capace di un flusso superiore ai $500 \mathrm{ml} / \mathrm{min}$ e di pompa della soluzione elettrolitica capace di un flusso superiore ai $700 \mathrm{ml} / \mathrm{min}$. La somministrazione del liquido di sostituzione viene effettuata con una pompa comandata a mano, controllando l'entità e la regolarità della somministrazione mediante pesata continua del paziente. $\grave{E}$ indispensabile, pertanto, essere provvisti di letto o poltrona bilancia con video display.

La seguente Figura mostra l'apparecchiatura per effettuare l'emodiafiltrazione ad alto flusso (Fig. 12).

Per evitare la manualità della somministrazione del liquido di sostituzione, sarebbe opportuno poter disporre di un monitor automatico che avesse la capacità di misurare momento per momento la quantità di emofiltrato più la quantità del liquido di dialisi che vengono allontanati attraverso la

TAB. II

\begin{tabular}{llcccc}
\hline Filtro & Membrana & $\begin{array}{c}\text { Ultrafiltra- } \\
\text { zione } \\
\text { media } \\
\mathrm{ml} / \mathrm{min}\end{array}$ & $\begin{array}{c}\text { Clearance } \\
\text { media urea } \\
\mathrm{ml} / \mathrm{min}\end{array}$ & $\begin{array}{c}\text { Clearance } \\
\text { media } \\
\text { creatinina } \\
\mathrm{ml} / \mathrm{min}\end{array}$ & $\begin{array}{c}\text { Clearance } \\
\text { media } \\
\text { fosfato } \\
\mathrm{ml} / \mathrm{min}\end{array}$ \\
\hline $\begin{array}{l}\text { Filtral Hospal } \\
\mathrm{m}^{2} 1.6\end{array}$ & $\begin{array}{l}\text { Poliacriloni- } \\
\text { trile }\end{array}$ & $146 \pm 12$ & $313 \pm 10$ & $256 \pm 10$ & $239 \pm 11$ \\
\hline $\begin{array}{l}\text { Pan 250 Asahi } \\
\mathrm{m}^{2} 1.8\end{array}$ & $\begin{array}{l}\text { Poliacriloni- } \\
\text { trile }\end{array}$ & $155 \pm 15$ & $335 \pm 11$ & $285 \pm 11$ & $245 \pm 14$ \\
\hline $\begin{array}{l}\text { Rapido Bellco } \\
\mathbf{m}^{2} 1.8\end{array}$ & Polisulfone & $160 \pm 14$ & $365 \pm 10$ & $302 \pm 11$ & $258 \pm 14$ \\
\hline $\begin{array}{l}\text { Hemoflow HF } 80 \\
\text { Fresenius }{ }^{2} 1.9\end{array}$ & Polisulfone & $165 \pm 16$ & $386 \pm 12$ & $297 \pm 10$ & $262 \pm 15$ \\
\hline $\begin{array}{l}\text { Filtryzer B1-2.0 } \\
\text { Toray }\end{array}$ & $\begin{array}{l}\text { Polimetilme- } \\
\text { tacrilato }\end{array}$ & $140 \pm 14$ & $348 \pm 13$ & $286 \pm 10$ & $237 \pm 11$ \\
\hline
\end{tabular}




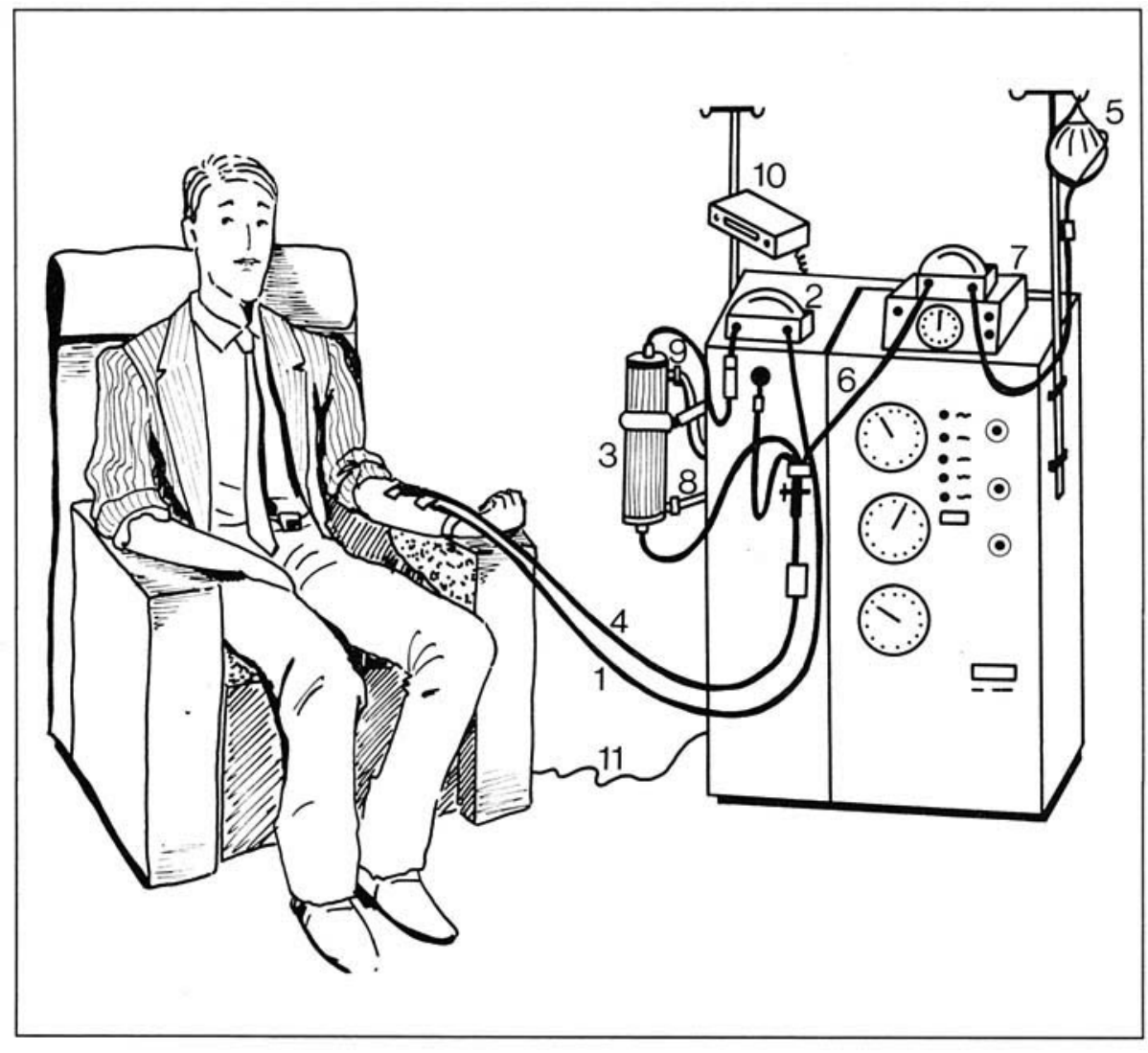

Fig. 12 - 1 Linea arteriosa - 2 Pompa sangue - 3 Filtro -4 Linea venosa -5 Liquido di sostituzione -6 Linea liquido di sostituzione -7 Pompa liquido di sostituzione -8 Ingresso soluzione elettrolitica - 9 Uscita liquido di dialisi ed emofiltrato - 10 Video display peso corporeo - 11 Collegamento elettronico poltrona bilancia-video display

medesima linea di scarico. Nello stesso tempo il monitor dovrebbe reinfondere al paziente, sempre istantaneamente, la quantità di liquido di sostituzione corrispondente all'emofiltrato meno la perdita di peso programmata.

Il monitor dovrebbe avere la capacità di adattarsi istante per istante alle prestazioni del filtro.

Quando abbiamo programmato il flusso ematico, il flusso della soluzione elettrolitica e la pressione transmembrana ed abbiamo stabilito quanto liquido di sostituzione dobbiamo somministrare, è l'efficienza del filtro che conduce il trattamento, che in base a questa efficienza, potrà avere una durata variabile. Non esistono monitors con queste caratteristiche. Quelli che sono in commercio vincolano alla programmazione della quantità di emofiltrato per minuto la possibile prestazione superiore del filtro, che non dovrebbe essere condizionata.

In alcuni pazienti non è possibile praticare l'emodiafiltrazione ad alto flusso a causa della scarsa portata dell'accesso vascolare. In genere però, un accesso vascolare correttamente eseguito ha una portata ematica nettamente superiore ai $500 \mathrm{ml} / \mathrm{min}$.

\section{Discussione}

Il grande vantaggio della emodiafiltrazione ad alto flusso è rappresentato dalla durata del singolo trattamento, che è nettamente inferiore a quella di qualsiasi altra me- todica depurativa. Infatti l'HDF è l'unico trattamento che consente di sfruttare contemporaneamente la clearance diffusiva a quella convettiva.

Nel seguente schema è rappresentata la durata media del singolo trattamento con le varie metodiche in un soggetto del peso di 70 $\mathrm{kg}$.

- Emodialisi convenzionale = 240 minuti

- Emodialisi ad alta efficienza $=$ 180 minuti

- Emofiltrazione $=240$ minuti

- Emodiafiltrazione ad alto flusso $=130$ minuti.

Per tutti i tipi di trattamento sostitutivo della funzione renale, la durata (t) della singola seduta si determina, per ciascun paziente, mediante il rapporto fra il presumibile volume di distribuzione dell'urea (V) e la clearance della stessa (K). Considerando che il volume di distribuzione è uguale a circa il $60 \%$ del peso corporeo, la durata media del singolo trattamento di emodiafiltrazione ad alto flusso per un soggetto di $70 \mathrm{~kg}$ sarà compresa fra 110 e 130 minuti a seconda del filtro che viene usato fra quelli indicati nella precedente Tabella. (Esempio: $60 \%$ di $70=$ $42.000 \mathrm{ml}$ che, diviso per la clearance dell'urea, ci fornisce il tempo di trattamento in minuti).

Tuttavia, per la emodiafiltrazione il tempo di trattamento non viene prestabilito, perché è più corretto procedere come verrà detto più dettagliatamente in seguito.

Riguardo all'interpretazione di $\mathrm{K}, \mathrm{t}$ e $\mathrm{V}$, dobbiamo precisare che:

$$
\text { se } \frac{V}{K}=t \text { e } K \cdot t=V, \frac{K \cdot t}{V}=1
$$

Quando l'indice $\frac{\text { K.t }}{V}$ risulta uguale 
o superiore a 1 un trattamento viene considerato adeguato. È proprio sulla rimozione di urea che oggi ci basiamo per giudicare l'adeguatezza di un trattamento.

La Tabella seguente mostra l'efficienza della emodialisi convenzionale, dell'emofiltrazione e dell'emodiafiltrazione ad alto flusso sulla rimozione di urea. (Tab. III).

Osservando la Tabella relativa alla efficienza sulla rimozione di urea, si può notare che il trattamento di emodialisi può essere adeguato o inadeguato perché l'indice K.t/V è sempre teorico poiché, comunemente, viene stabilito un tempo di circa 240 minuti per tutti i pazienti. Per essere sicuri di ottenere un K.t/ $\mathrm{V}$ uguale o superiore a 1 , bisognerebbe che la clearance dell'urea fosse sempre la stessa con lo stesso filtro.

Purtroppo, per cause varie, lo stesso tipo di filtro non fornisce la stessa clearance ad ogni trattamento.

Quindi, anche assumendo che il $V$ sia esatto, manca l'esattezza del $K$ (a meno che non si pratichi la clearance dell'urea ad ogni seduta) e quindi anche del $\mathbf{t}$.

Tuttavia, ai fini pratici, con le varie gamme di filtri oggi a disposizione, 240 minuti di trattamento sono sufficienti per qualsiasi paziente.

L'Emofiltrazione garantisce un trattamento adeguato se viene emofiltrata una quantità di liquido uguale al volume di acqua corporea del paziente ed alla sua perdita di peso programmata. Tuttavia, anche con i più validi emofiltri che possono consentire un emofiltrato medio di oltre $160 \mathrm{ml} / \mathrm{min}$, la durata della singola seduta di emofiltrazione è sempre compresa fra $i$ 200 e i 250 minuti, a seconda del peso corporeo del paziente e quindi del suo volume di acqua corporea.

L'Emodiafiltrazione ad alto flusso assicura sempre un trattamento sicuramente adeguato in un tempo che è circa la metà rispetto a quello necessario per la emodialisi e per la emofiltrazione.

Come per la emofiltrazione, anche per la emodiafiltrazione ad alto flusso la durata del singolo trattamento non è prestabilita, ma è quella necessaria ad emofiltrare una quantità di liquido, che in questo caso è uguale alla metà del volume di acqua corporea del paziente, più la perdita di peso programmata. Infatti, dobbiamo considerare che nello stesso tempo in cui durante l'emodiafiltrazione av- viene questa emofiltrazione, (che possiamo definire clearance convettiva dell'urea) avviene una depurazione diffusiva (clearance diffusiva) che è sempre superiore alla metà del volume di distribuzione dell'urea, poiché con tutti i filtri particolarmente idonei a praticare l'emodiafiltrazione ad alto flusso, la depurazione diffusiva è sempre superiore a quella convettiva.

Il tempo del singolo trattamento può essere più o meno lungo e dipende dalla prestazione del filtro.

Se, invece del tempo, prestabiliamo la quantità di liquido che deve essere emofiltrata dall'inizio alla fine del trattamento, siamo sicuri di praticare una emodiafiltrazione adeguata.

Se per qualsiasi motivo inerente la prestazione del filtro, diminuisce $\mathrm{K}$, aumenterà $\mathrm{t}$; se diminuisce $\mathrm{t}$ significa che è aumentato $\mathrm{K}$. Pertanto, il trattamento risulterà sempre della stessa efficienza.

Dal punto di vista pratico, è importante considerare che la emodiafiltrazione ad alta efficienza ci consente di trattare, nello stesso tempo necessario alla emodialisi convenzionale, un numero doppio di pazienti con lo stesso personale medico e infermieristico.

TAB. III

\begin{tabular}{llll}
\hline & $\begin{array}{c}\text { Durata media della singola } \\
\text { seduta }\end{array}$ & Kt/V & $\begin{array}{c}\text { Valutazione del } \\
\text { trattamento }\end{array}$ \\
\hline $\begin{array}{l}\text { Emodialisi } \\
\text { convenzionale }\end{array}$ & 240 minuti & adeguato o \\
Emofiltrazione & $\begin{array}{l}\text { quella necessaria a emofiltrare } \\
\text { una quantità di liquido uguale } \\
\text { al volume di acqua corporea } \\
\text { più la perdita di peso } \\
\text { programmata }\end{array}$ & adeguato \\
\hline $\begin{array}{l}\text { Emodiafiltrazione } \\
\text { ad alto flusso }\end{array}$ & $\begin{array}{l}\text { quella necessaria a emofiltrare } \\
\text { una quantità di liquido uguale } \\
\text { alla metà del volume di acqua } \\
\text { corporea più la perdita di peso } \\
\text { programmata }\end{array}$ & $>1$ & adeguato \\
\hline
\end{tabular}

